\title{
Optical Technology Options for Programmable S-BVT
}

\author{
Michela Svaluto Moreolo, Senior Member, IEEE, Josep M. Fàbrega, Senior Member, IEEE, Laia Nadal, \\ and Laura Martín \\ Centre Tecnolòic de Telecomunicacions de Catalunya (CTTC/CERCA), Castelldefels (Barcelona), Spain \\ e-mail:michela.svaluto@cttc.es
}

\begin{abstract}
Optical technology options for programmable sliceable bandwidth/bitrate variable transceivers (S-BVT) are presented, reviewing our proposals based on adaptive multicarrier modulation. Special attention will be devoted to data plane solutions with high degree of (re)configurability and flexibility for the optical metro network segment. We propose transceiver architectures based on modular design in order to target grow-as-needed approach and optical domain disaggregation, identifying data plane elements that can be considered as separate management and functional entities, as well as functionalities that can be moved to software.

Our work in this direction is presented, focusing on the S-BVT features and capabilities, taken into account the optical system and its interrelationship with other elements of the network.

Keywords: Sliceable bandwidth/bitrate variable transceiver (S-BVT), multicarrier modulation (MCM), flexigrid, disaggregated optical networks.
\end{abstract}

\section{INTRODUCTION}

Programmable transceivers are key enablers for future optical networks, and particularly for the metro segment, which represents the most challenging scenario to be addressed [1]. Indeed, we are witnessing a fast growing of the speed and volume of traffic driven by novel and broadband $(5 \mathrm{G})$ services. This affects all network segments, but it is particularly relevant and most prominent in metro networks. For example, according to CISCO reporting, the $35 \%$ of end-user Internet traffic will be delivered within a metro network by 2021 (being $22 \%$ in 2016) [2]. The need for managing adaptive bit rates and high peak-average traffic ratio, according to the available bandwidth and network resources, while minimizing the cost per bit, the power consumption and the footprint, is posing several technological challenges that have to be addressed. Furthermore, in this evolutionary scenario, the scalability of any technological solution and its interoperability with the rest of the system, including analogue and digital domains, hardware and software elements, for a suitable data and control plane implementation, are open issues currently discussed and object of study and analysis.

In this context, the adoption of programmable transceivers results attractive to enable the softwarization and intelligent control of data plane, as well as a soft-migration towards a more flexible paradigm [3, 4]. Furthermore, a modular design facilitates the scalability and a suitable sizing of the solution according to the network segment or specific node requirements, promoting a grow/pay-as-you-need approach. This also paves the way towards optical network disaggregation, while fostering interoperability $[5,6]$.

In particular, a sliceable bandwidth/bitrate variable transceiver (S-BVT), composed of multiple modules based on multicarrier modulation (MCM), enables to support flexible and programmable multi-flow, multi-rate, multiformat and multi-reach transmission. The number of modules can be increased in order to improve the capacity and the potential of the S-BVT to adapt to the network requirements in a grow-as-needed approach.

The S-BVT configurations reported in this work are characterized by a high degree of reconfigurability/flexibility, enabling spectral manipulation with super-wavelength granularity in the optical/analogue domain and also sub-wavelength granularity in the electrical/digital domain. These and other aspects will be further detailed in Sec. 2, where the description of a programmable S-BVT based on adaptive MCM is provided and the related technology options discussed. Our recent results on the different transceiver configurations/options are provided in Sec. 3, taking into account the system and other network elements towards an optimized design, particularly targeting optical metro networks and future optical disaggregation.

\section{PROGRAMMABLE S-BVT BASED ON ADAPTIVE MCM}

Figure 1 shows an example of an S-BVT with $N$ modules. Each module can be enabled or disabled according to the network needs and it is based on MCM with receiver configuration adopting direct detection (DD).

The S-BVT generates up to $N$ flows/slices with cost-effective optoelectronic subsystems. Particularly, the bandwidth/bitrate variable transmitter (BVTx) optoelectronic front-end consists of a simple Mach-Zehnder modulator (MZM), driven by a tunable laser source (TLS) for arbitrary wavelength selection, and the DD bandwidth/bitrate variable receiver (BVRx) requires a simple photo-detector (PIN) with transimpedance amplifier (TIA).

The multiple flows are optically aggregated/distributed by using a bandwidth variable (BV) wavelength selective switch (WSS), which can be implemented in liquid crystal on silicon (LCoS) technology. This element can be also suitably programmed/configured to serve as i) filter bank to enable single sideband (SSB) modulation of each generated flow/slice; or/and ii) optical orthogonal transform processor for superchannel 
generation [7]. SSB-OFDM allows extending the achievable reach thanks to its higher robustness against chromatic dispersion with respect to DMT, which in turn is more cost-effective and does not require any additional filtering stage [4]. DD can be replaced by coherent (CO) detection for improving the performance.

At each BVT module, the adaptive digital signal processing (DSP) supports MCM, either DMT or OFDM, and a multiple format mapper, as detailed in [4]. The mapper enables uniform loading (UL) of the digital/electrical DMT/OFDM subcarriers or bit and power loading (BL/PL) algorithm, using adaptive modulation formats including BPSK and $M$-QAM (with $M=2 n$ and $2 \leq n \leq 8$ ), for achieving multiple rate/reach. The sub-wavelength granularity is defined as the ratio between the slice/flow bandwidth (BW) and the number of electrical/digital DMT/OFDM subcarriers $\left(N_{s u b c}\right)$. For example, considering a BW of $20 \mathrm{GHz}$ and $256 \mathrm{OFDM}$ subcarriers, the subcarrier spacing and thus the minimum sub-wavelength granularity result about $80 \mathrm{MHz}$.

The S-BVT, by means of an S-BVT agent, allows the SDN controller to configure variable parameters in both the digital and analogue domains, such as the number of enabled slices, the occupied frequency slots (i.e. the bandwidth occupancy), the aggregator transfer function per port, etc. (see table on the right of Fig. 2). With respect to the digital part, by suitably selecting the DSP mode, it is possible to activate the transmission of uniformly loaded probing signals. This allows retrieving the channel state information (CSI), by means of calculating the signal-to-noise ratio (SNR) per each subcarrier. Accordingly, as illustrated in Fig. 1, the subcarrier loading is adapted to achieve the successful data flow transmission at a target bit rate (in margin adaptive, MA, mode) or at a target performance (in rate adaptive, RA, mode), which requires to select the suitable gap. The BER over the established path (target reach) is calculated at the BVRx DSP. Different FEC options, either hard-decision (HD) or soft-decision (SD), and the related overhead (e.g. 7\% or 20\%) - varying the total overhead and thus the achievable net bit rate - are taken into account to target a certain BER (e.g. 10 ${ }^{-3}$, $4.62 \cdot 10^{-3}$, or $2 \cdot 10^{-2}$ ). For the digital-to-analogue conversion (DAC) and analogue-to-digital conversion (ADC), the sample rate can vary according to the available device (e.g. $64 \mathrm{GSa} / \mathrm{s})$. Depending on the adopted element the signal bandwidth could be affected/limited.

The programmable elements at the optoelectronic front-end of the BVT module include the TLS for enabling/disabling the transmission of each flow/slice and the corresponding wavelength and power setting. Also the photo-detectors at the receiver can be enabled/disabled, accordingly. The external modulator (MZM) bias can be also suitably selected for working at the quadrature or close by the null point.

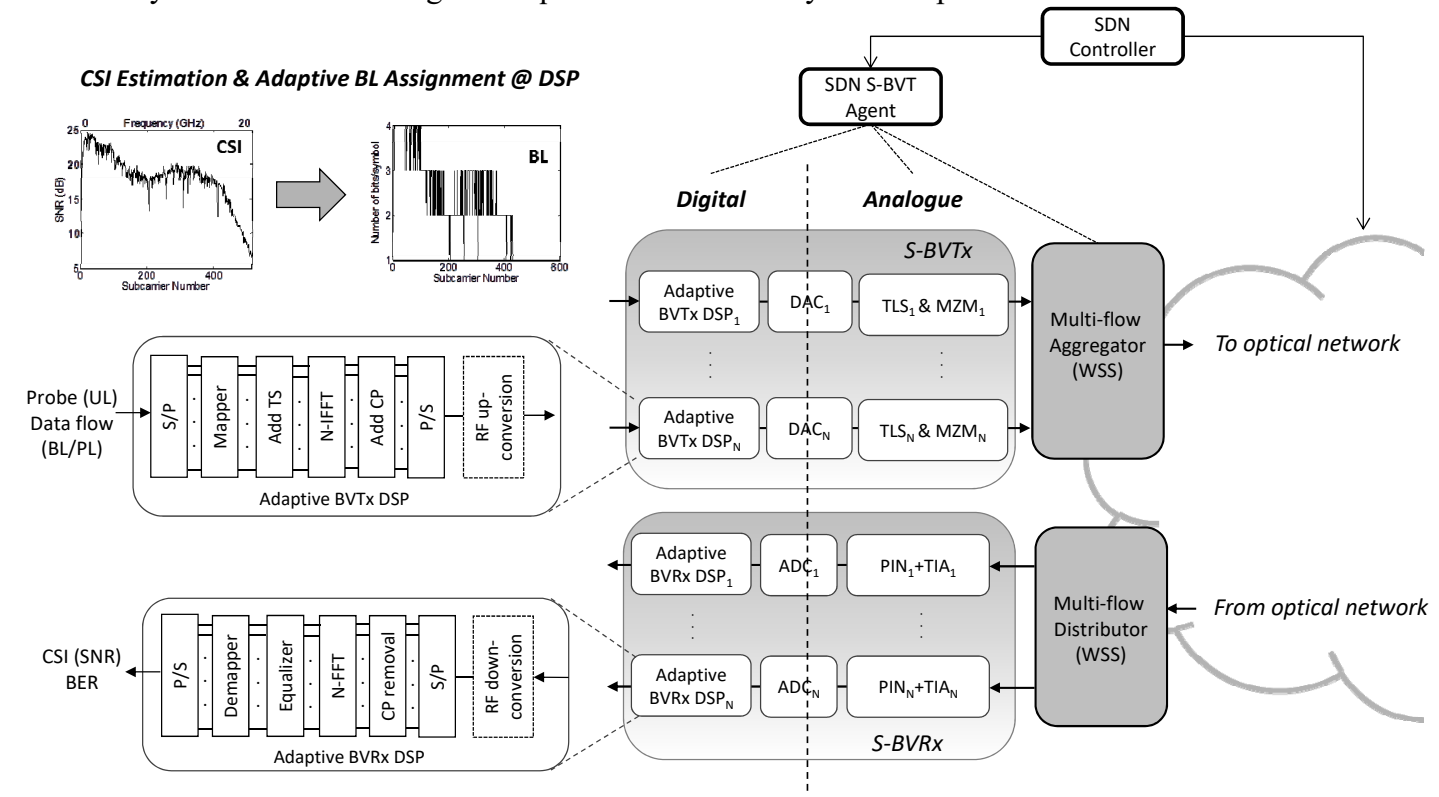

Figure 1. S-BVT based on MCM (DMT/OFDM) adopting external modulation and DD. In the insets, the adaptive DSP at the BVTx and BVRx are detailed and an example of BL assignment is reported.

\subsection{Technology options}

The suitable transceiver technology to be adopted depends on the targeted network segment and application. The fundamental S-BVT blocks and/or elements can be selected according to their cost/complexity and achievable performance, identifying different flexibility level/requirements and transceiver configurations. Figure 2 (table on the left-hand side) summarizes them for a generic S-BVT based on MCM.

For example, transceivers adopting $\mathrm{CO}$ detection provide flexibility and high capacity for an extended achievable reach [3], but are more complex and costly than the options based on DD. Particularly for optical metro networks, a reduction of the transceiver cost is highly desirable. In fact, this network segment poses several challenges in terms of traffic dynamicity, cost and energy consumption [2]. 
For the S-BVT based on MCM, we mainly identify three configurations: 1) DMT intensity modulation with DD, 2) SSB-OFDM with DD, and 3) DMT (amplitude modulation) or OFDM with CO receiver. The first option is the simplest with the lowest cost/complexity at the expenses of the performance and spectral efficiency (SE), since the double of BW occupation is required compared to options 2 and 3. To further reduce the transmitter cost, also direct laser modulation can be envisioned. In this case, vertical cavity surface emitting laser (VCSEL) technology can be adopted to dramatically reduce the cost, power consumption and footprint of the solution [8]. However, in this work, we focus on external modulation with TLS. The second option combines SSB-OFDM with DD and allows extending the achievable reach, since it is more robust against chromatic dispersion. The ultimate performance can be achieved by the third configuration adopting $\mathrm{CO}$ receiver (with tunable local oscillator, TLO), at the expenses of higher cost and complexity at the receiver with respect to the other options.

We propose that the DSP block is adaptive, implementing BL and PL algorithm to guarantee more flexibility and better performance than a simple uniform assignment of bits per subcarrier. UL is used only to retrieve the CSI and not for transmission mode operation. The availability of high-speed and large bandwidth DAC/ADC facilitates the improvement of the capacity and performance of the transceiver, without introducing severe limitation on the bandwidth (which we assume that is not affected by the optoelectronic front-ends). The aggregator/distributor can be a programmable and BV WSS, implemented with LCoS technology, or can be a simple and cost-effective passive element, implemented for example in planar lightwave circuit (PLC). The costeffectiveness of this last option limits the flexibility and programmability of the transceiver architecture.

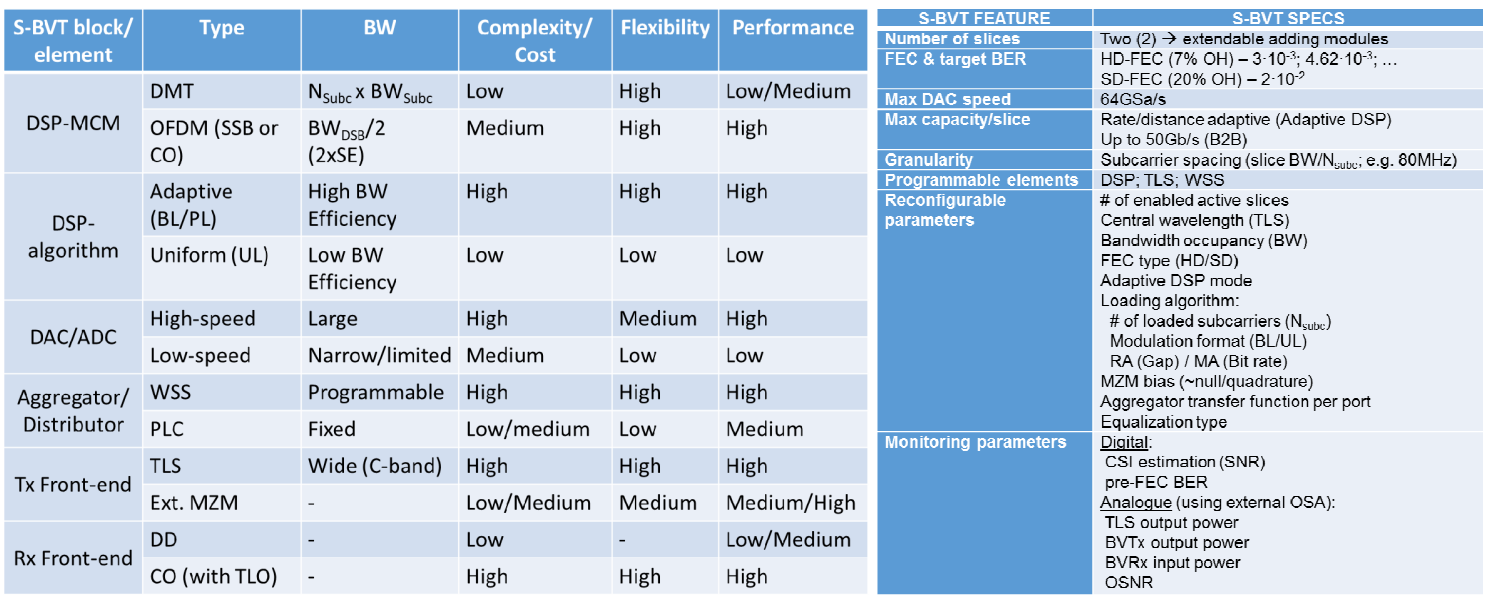

Figure 2. S-BVT based on MCM block/elements, features and specs.

Figure 2 also reports the features and specs of an S-BVT based on MCM being developed within the EOS platform of the CTTC ADRENALINE testbed. There, the reconfigurable and monitoring parameters are listed. The electrical/optical performance monitoring is achieved by means of a synergic interworking among data plane elements and control plane. Particularly, the digital parameters of the S-BVT that can be monitored per each flow/slice are i) the SNR of each digital/electrical DMT/OFDM subcarrier, providing the CSI, and ii) the (pre-FEC) BER. It is important to remark the self-performance monitoring capability of MCM techniques [4].

Monitoring parameters of the analogue optical system can be the TLS output power, the BVTx output power and the BVRx input power. It is worth mentioning that these parameters can be monitored with the aid of a data plane element external to the S-BVT (e.g. optical spectrum analyser, OSA).

\section{RESULTS AND DISCUSSION}

Different implementations of the proposed S-BVT configurations have been experimentally assessed in back-toback (B2B) and within the 4-node ADRENALINE network testbed [1, 4, and 7]. It has been found that a single slice can support a maximum capacity of $50 \mathrm{~Gb} / \mathrm{s}$, considering a $20 \mathrm{GHz}$ signal $\mathrm{BW}$ and adopting a BVT configuration with SSB-OFDM and DD. The adaptive loading is implemented at the DSP, the SSB is performed at the WSS and a DAC at $64 \mathrm{GSa} / \mathrm{s}$ is required (see the specs reported in Fig. 2); the ADC is implemented by adopting an oscilloscope with maximum sample rate of $100 \mathrm{GSa} / \mathrm{s}$. An example of BL assignment is shown in Fig. 1, considering the CSI retrieved at the BVRx after transmission of a 512-subarrier OFDM signal with UL (4-QAM) over a targeted path of the 4-node ADRENALINE network. This transceiver configuration arises as flexible and adaptive candidate for designing SDN-enabled S-BVT architecture suitable for future optical metro networks. Thus, considering the evolutionary scenario of metro networks, it has been also assessed in the context of flexi-grid paradigm. In particular, the filter narrowing effect has been studied for low bandwidth/bitrate $(10 \mathrm{~Gb} / \mathrm{s})$ connections, considering the concatenation of up to 12 filters (two filters per node), with different filter bandwidths: $12.5 \mathrm{GHz}, 25 \mathrm{GHz}$, and $50 \mathrm{GHz}$. Significant signal degradation for filter bandwidths of $12.5 \mathrm{GHz}$ with 
respect to $25 \mathrm{GHz}$ and $50 \mathrm{GHz}$ have been evidenced [9]. Thus, the interrelationship with other network elements should be taken into account for an optimized design, especially when dealing with low bandwidth connections.

A more cost-effective BVT configuration adopting DMT with lower speed DAC (e.g. $24 \mathrm{GSa} / \mathrm{s}$ or $28 \mathrm{GSa} / \mathrm{s}$ ) and DD can be considered for flexible/adaptive transmission in an optical metro network adopting for example lossless SOA-based switching nodes [10].

In [7], we have proposed and experimentally assessed a hybrid and electro-optical MCM scheme exploiting super/sub-wavelength granularity for flexible/efficient multiple-flow transmission within elastic optical metro networks. Specifically, multiple OFDM electrical signals/flows are multiplexed into superchannels by optically implementing the discrete wavelet packet transform (DWPT) and its inverse to increase the S-BVT spectral efficiency. In fact, the multiple flows can be packed with no guard-band and unlike the fast Fourier transform (FFT), the wavelet finite bases functions (with time and frequency localization) enabling simpler implementation. It has been proven that this S-BVT scheme enables to pack multiple flows at $25 \mathrm{~Gb} / \mathrm{s}$ each (in B2B), adopting a transceiver configuration based on DMT (10 GHz bandwidth and sample rate of $20 \mathrm{GSa} / \mathrm{s}$ ) with external MZM (biased at the quadrature point) and TLS combined with DD. Also a configuration based on amplitude modulation (MZM biased close by the null point) and CO-BVRx has been envisioned to improve the capacity/reach performance of the transceiver. The assessment has been also performed in the ADRENALINE testbed, showing that up to $120 \mathrm{~km}$ and 3-hop path can be successfully covered [7]. The WSS can be suitably programmed by selecting the appropriate attenuation/phase of each port to set the transfer function corresponding to the resulting concatenated wavelet filtering stage. Good matching of performance has been observed for two flows, implementing the Haar wavelet with the WSS or either a simple coupler. Actually, the aggregator can be implemented using Mach-Zehnder interferometers integrated employing silicon-on-insulator technology, to further enhance the solution power efficiency at the expense of the system programmability and flexibility (as mentioned in Sec. 2.1). Control aspects have been analyzed, including the development of a YANG model and the configuration of the S-BVT and its slices, as shown in [7]. The development of open data models is crucial for disaggregated optical networks and to move towards the implementation of white boxes [6].

\section{CONCLUSIONS}

Programmable transceivers are key enabler for addressing the challenges of future optical metro networks and for a smooth migration towards flexible, open and programmable paradigms. In this work, we have presented technology options and possible configurations of S-BVT based on MCM that we consider suitable candidate to target grow-as-needed approach and optical domain disaggregation, identifying the fundamental elements in the digital and analogue domains, their features and related flexibility, reconfigurability and monitoring capabilities. Recent results have been reported, taken into account the optical system and the interrelationship of the S-BVT with other elements of the network.

\section{ACKNOWLEDGEMENTS}

This work has been supported by the EU-H2020-ICT-2016-2 Metro-Haul Project (G.A. 761727) and the Spanish DESTELLO project (TEC2015-69256-R).

\section{REFERENCES}

[1] M. Svaluto Moreolo, J. M. Fabrega, and L. Nadal: S-BVT for next-generation optical metro networks: benefits, design and key enabling technologies, in Proc. SPIE 10129, San Francisco, CA (USA), Jan. 2017.

[2] CISCO White Paper: "The Zettabyte Era: Trends and Analysis," June 2017

[3] N. Sambo, et al:: Next generation sliceable bandwidth variable transponder, IEEE Communications Magazine, vol. 53, no. 2, pp. 163-171, Feb. 2015.

[4] M. Svaluto Moreolo, et al:: SDN-Enabled Sliceable BVT Based on Multicarrier Technology for Multiflow Rate/Distance and Grid Adaptation, J. Lightwave Technol., vol. 34, no. 6, pp. 1516-1522, March 2016.

[5] M. De Leenheer, et al.: Open and Programmable Metro Networks, in Proc. OFC 2016, paper Th1A.7.

[6] E. Riccardi, P. Gunning, O. Gonzalez de Dios, M. Quagliotti, V. Lopez and A. Lord: An Operator's view on introduction of White Boxes in Optical Networks, J. Lightwave Technol., Early Access, March 2018.

[7] L. Nadal, et al.: Programmable SDN-enabled S-BVT based on hybrid electro-optical MCM, J. Opt. Commun. Netw., vol. 10, no. 6, pp. 593-602, June 2018.

[8] M. Svaluto Moreolo, J. M. Fàbrega, L. Nadal, F. J. Vílchez: Exploring the Potential of VCSEL Technology for Agile and High Capacity Optical Metro Networks, in Proc. ONDM 2018, Dublin (Ireland), May 2018.

[9] L. Martin, J. M. Fabrega, M. Svaluto Moreolo, F. J. Vilchez, L. Nadal: Experimental Assessment of Filter Narrowing Effect for Low Bandwidth Connections in EON, IEEE Photonics Technology Letters, vol. 29, no. 23, pp. 2027-2030, Dec. 2017.

[10] N. Calabretta, et al:: SOA based photonic integrated WDM crossconnects for optical metro-access networks, Applied Sciences, vol. 9, no. 7, p. 865, 2017. 